\title{
Speckled Oral Leukoplakia
}

National Cancer Institute

\section{Source}

National Cancer Institute. Speckled Oral Leukoplakia. NCI Thesaurus. Code C4695.

Non-homogenous leukoplakia on the oral mucosa. It is associated with a high risk for malignant transformation. 